\title{
Diversity of $A T M$ gene variants: a population-based genome data analysis for precision medicine
}

\author{
Hisanori Fukunaga ${ }^{1,2,3^{*}}$ D, Yasuyuki Taki ${ }^{3,4}$ and Kevin M. Prise
}

\begin{abstract}
Background: Ataxia-telangiectasia (AT) is a rare autosomal recessive disorder that causes deficiency or dysfunction of the ataxia-telangiectasia mutated (ATM) protein. Not only AT patients, but also certain ATM heterozygous mutation carriers show a significantly reduced life expectancy due to cancer and ischemic heart disease; in particular, female carriers having particular alleles have an increased risk of breast cancer. The frequency of such risk heterozygotes at a population level remains to be fully determined, and evidence-based preventive medical guidelines have not yet been established.

Methods: Using the 3.5KJPNv2 allele frequency panel of Japanese Multi Omics Reference Panel v201902, which shows single-nucleotide variant (SNV) and insertion/deletion (INDEL) allele frequencies from 3552 Japanese healthy individuals, we investigated the diversity of ATM gene variants.

Results: We detected 2845 (2370 SNV and 475 INDEL) variants in the ATM gene, including 1338 (1160 SNV and 178 INDEL) novel variants. Also, we found a stop-gained SNV (NC_000008.11:g.108115650G > A (p.Trp266*)) and a disruptive-inframe-deletion (NC_000008.11:g. 108181014AAGAAAAGTATGGATGATCAAG/A (p.Ala1945_ Phe1952delinsVal) and two frameshift INDELs (NC_000008.11:g.108119714CAA/C (p.Glu376fs) and NC_000008.11: g.108203577CTTATA/C (p.lle2629fs)), which would be novel variants predicted to lead to loss of ATM functionality. Conclusion: The combination of population-based biobanking and human genomics provided a novel insight of diversity of ATM gene variants at a population level. For the advancement of precision medicine, such approach will be useful to predict novel pathogenic/likely pathogenic variants in the ATM gene and to establish preventive medical guidelines for certain ATM heterozygotes pertaining to their risk of particular diseases.
\end{abstract}

Keywords: Ataxia-telangiectasia mutated, Population-based biobank, Heterozygotes, Precision medicine, Wholegenome reference panel

\section{Introduction}

Ataxia-telangiectasia (AT) is a rare autosomal recessive disorder clinically characterized by cerebellar degeneration, immune-deficiency, radiation hypersensitivity, and cancer predisposition. Also, metabolic disorders implicated in cardiovascular and liver diseases are frequently observed in adolescent AT patients [1]. The worldwide

\footnotetext{
* Correspondence: hfukunaga01@qub.ac.uk

${ }^{1}$ Centre for Cancer Research and Cell Biology, Queen's University Belfast, 97 Lisburn Road, Belfast BT9 7AE, UK

${ }^{2}$ Shonan Kamakura General Hospital, 1370-1 Okamoto, Kamakura, Kanagawa 247-8533, Japan

Full list of author information is available at the end of the article
}

prevalence of AT is estimated to be between 1 in 40,000 and 1 in 100,000 live births [2].

The ataxia-telangiectasia mutated (ATM) gene was first reported in 1995 as the causative gene responsible for AT [3]. Most AT patients lack functional ATM protein due to missense or non-sense mutations in the ATM gene, which result in truncated or unstable ATM variants [4]. The ATM gene is located on chromosome 11q22-q23, spanning approximately $150 \mathrm{~kb}$ of genomic DNA, and encodes a protein of 3056 amino acids which is a phosphoinositidyl 3-kinase (PI3K)-family kinase [5]. ATM forms dimers or oligomers under non-stress conditions, and DNA damage induces intermolecular autophosphorylation of Ser1981

(C) The Author(s). 2019 Open Access This article is distributed under the terms of the Creative Commons Attribution 4.0 International License (http://creativecommons.org/licenses/by/4.0/), which permits unrestricted use, distribution, and 
that causes dimer dissociation and initiates intracellular ATM kinase activity [6]. In addition to Ser1981, other autophosphorylation sites, Ser367, Ser1893, and Ser2996 are physiologically important parts of the DNA damage response $[7,8]$. Also, ATM phosphorylates several proteins involved in cell cycle checkpoint control, apoptosis, and DNA repair, including p53, Chk2, BRCA1, RPAp34, H2AX, SMC1, FANCD2, Rad17, Artemis, and Nbs1 [9].

Not only AT patients, but also certain ATM heterozygous mutation carriers have a reduced life expectancy. In 1987, Swift and co-workers reported that for heterozygotes responsible for $\mathrm{AT}$, the relative risk of cancer was estimated to be 2.3 for men and 3.1 for women [10]. Furthermore, according to a systematic review in 2016, siblings of AT patients who are heterozygous carriers of pathogenic ATM gene mutations have a significantly increased risk of cancer and ischemic heart disease (relative risk (RR) 1.7, 95\% confidential interval (CI) 1.2-2.4), and female heterozygotes having particular alleles have an increased risk of breast cancer (RRwomen 3.0, 95\% CI 2.1-4.5) [11]; thus, certain ATM heterozygous mutation carriers should be made aware of lifestyle factors that contribute to the development of such diseases. However, the accurate frequency of heterozygous carriers of pathogenic mutations in the ATM gene at a population level, to date, remains to be determined, and evidence-based preventive medical guidelines have not yet been established [12]. From the point of view of precision medicine, appropriate approaches are immediately needed for $A T M$ heterozygotes pertaining to their risk of particular diseases.

\section{Methods}

To address the issue, we used the 3.5KJPNv2 allele frequency panel [13-16], which is a part of Japanese Multi Omics Reference Panel version 201902 (Feb 2019, https:// jmorp.megabank.tohoku.ac.jp/201902/) released from the Tohoku University's Tohoku Medical Megabank Organization (ToMMo) [17]. This population-based wholegenome reference panel shows single-nucleotide variant (SNV) and insertion/deletion (INDEL) allele frequencies from 3552 Japanese healthy individuals. jMorp was originally published in 2018, as a multi-omics database of metabolites and proteins in plasma obtained from volunteers in ToMMo [17]. From jMorp release 201806 (Jun 2018, https://jmorp. megabank.tohoku.ac.jp/201806/), genomic variant data have been added, and the version 201,902 (Feb 2019, https://jmorp.megabank.tohoku.ac.jp/201902/) is where allele frequencies of all the genomic variants can be examined through the web interface.

Although they previously used an original re-sequencing workflow for the 1KJPN5 [13], 2KJPN, and 3.5KJPNv1, for building the 3.5KJPNv2, ToMMo decided to use a more common pipeline including the 1000 Genomes Project
[18] and gnomAD [19] algorithms to reduce technical biases and to allow comparisons to other populations. ToMMo customized three steps in the Genome Analysis Toolkit (GATK) Best Practices workflow, which are widely used in large-scale sequencing projects and recommend post-alignment processing before variant calling [20]: (1) the choice of the reference genome, (2) the use of base quality score recalibration (BQSR), and (3) the joint genotyping step. Although the GATK Best Practices workflow recommends that the BQSR step be carried out after the mapping, ToMMo did not do so, but checked concordance among two kinds of genotyping results: (i) genotyping results obtained after the incorporation of BQSR and (ii) results obtained without BQSR. Thus, the allele frequency panel, 3.5KJPNv2 of jMorp version 201902, can be easily used to compare the allele frequencies of different populations with the web interface.

The 3.5KJPNv2 is available at jMorp website, and the raw data in Variant Call Format (VCF) format was also registered at the NBDC Human Database (https:// humandbs.biosciencedbc.jp/en/) with accession code hum0015.v3 by the National Bioscience Database Center (NBDC) of the Japan Science and Technology Agency to ensure accessibility, preservation, and stability of the $3.5 \mathrm{KJPNv} 2$ datasets [16]. Individual's sequence data and genotyping results from which allele frequency dataset is constructed and validated are available upon request after approval of the Ethical Committee and the Materials and Information Distribution Review Committee of ToMMo.

In the present study, we accessed the raw data of the $3.5 \mathrm{KJPNv} 2$ allele frequency panel and investigated the allele frequencies of ATM gene variations, with the assumption of the Hardy-Weinberg equilibrium. Also, we investigated novel variants, which are predicted to lead to loss of ATM functionality based on their protein structures.

\section{Results and discussion}

As shown in Fig. 1a, with the 3.5KJPNv2 allele frequency panel, we searched for the allele frequencies of $A T M$ gene variations and found 2845 (2370 SNV and 475 INDEL) variants in the given population, including 1338 (1160 SNV and 178 INDEL) novel variants, which have not yet been assigned reference single-nucleotide polymorphism (SNP) ID numbers [21]. This result demonstrates that, with the assumption of the Hardy-Weinberg equilibrium, a large number of healthy individuals have novel heterozygous variants in the ATM gene, indicating that the $A T M$ genetic diversity is greater than expected.

We found a novel stop-gained SNV in the ATM gene, NC_000008.11:g.108115650G > A (p.Trp266*). As shown in Fig. 1b, this SNV removes all the main identified domains including the nuclear localization signal (NLS; aa 385 to 388), the leucine zipper (LZ; aa 1216 to 1241), the FRAP/ATM/TRRAP (FAT; aa 1960 to 2566), the kinase 


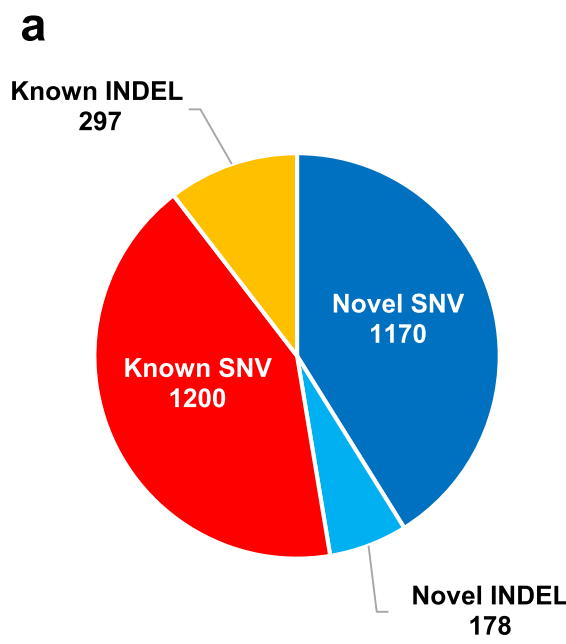

b

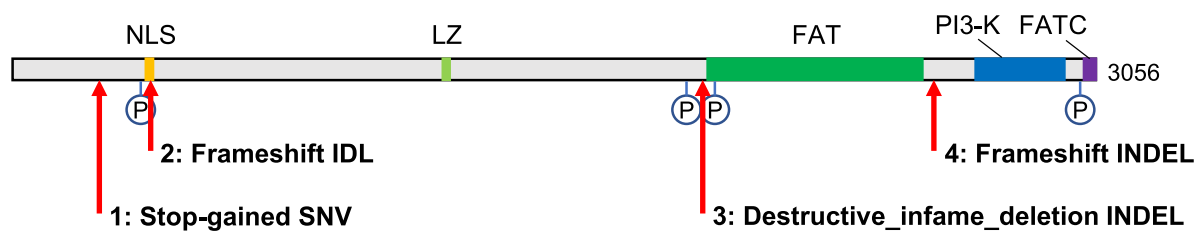

Fig. 1 Novel variants predicted to lead to loss of ATM functionality. a A total of 2845 ( 2370 SNV and 475 INDEL) variants in the ATM gene has confirmed, including 1338 (1160 SNV and 178 INDEL) novel variants which have not yet been assigned reference SNP ID numbers. b ATM is a protein of 3056 amino acids. The phosphorylation sites (P) indicate the positions of serine residues, Ser367, Ser1893, Ser1981, and Ser2996 [6-8]. The NLS ( aa 385 to 388), the LZ ( aa 1216 to 1241), the FAT ( aa 1960 to 2566), the PI3-K ( aa 2712 to 2962), and the FATC ( aa 3024 to 3056) domains are shown in orange, light green, green, blue, and violet respectively [9]. Novel variants predicted to lead to loss of ATM functionality are indicated using red arrows: (1) stop-gained SNV, NC_000008.11:g.108115650G > A (p.Trp266*), (2) frameshift INDEL, NC_000008.11:g.108119714CAA/C (p.Glu376fs), (3) disruptive-inframe-deletion INDEL, NC_000008.11:g.108181014AAGAAAAGTATGGATGATCAAG/A (p.Ala1945_Phe1952delinsVal), (4) frameshift INDEL, NC_000008.11:g.108203577CTTATA/C (p.lle2629fs)

(PI3-K; aa 2712 to 2962), and the FAT c-terminal (FATC; aa 3024 to 3056) domains, which are physiologically important for the activation and regulation of ATM kinase activity [9]. Also, this removes autophosphorylation sites, Ser367, Ser1893, Ser1981, and Ser2996, which are significantly related to ATM protein functionality. Taken together, the stop-gained SNV in the ATM gene is predicted to be a novel loss-of-function variant.

Next, we detected a novel disruptive_inframe_deletion, NC_000008.11:g.108181014AAGAAAAGTATGGATGATCAAG/A (p.Ala1945_Phe1952delinsVal), and two frameshift INDELs, NC_000008.11:g.108119714CAA/C (p.Glu376fs) and NC_000008.11:g.108203577CTTATA/C (p.Ile2629fs). Because of the dramatic changes of amino acid sequence and three-dimensional structure, all the INDELs are predicted to lead to loss of ATM functionality. Even NC_ 000008.11:g.108203577CTTATA/C, although maintaining more than 2500 normal amino acid sequence from the $\mathrm{N}$-terminal of ATM, removes normal C-terminal PI3-K domain and autophosphorylation site Ser2996, which are essential for ATM signaling in human cells.
To our knowledge, there is no previous study relating to the clinical risk of these four variants. However, according to the possible ATM functionality based on their protein structures, certain ATM heterozygous mutation carriers having these variants are likely to have a reduced life expectancy. From the point of view of precision medicine, personalized preventive medical strategies would be immediately needed.

\section{Conclusion}

In this study, we showed the diversity of ATM gene variants at a population level and found four novel variants which are predicted to lead to loss of ATM functionality. Further advancements in the combination of populationbased biobanking and human genomics are expected to further uncover the genetic basis of AT patients and certain ATM heterozygotes who have a reduced life expectancy. Also, for the advancement of precision medicine, such approach will be useful to establish an evidencebased guideline not only for AT patients but also for risk ATM heterozygotes seeking preventive medical strategies. 


\section{Abbreviations}

AT: Ataxia-telangiectasia; ATM: Ataxia-telangiectasia mutated; BQSR: Base quality score recalibration; Cl: Confidential interval; DNA: Deoxyribonucleic acid; FAT: FRAP/ATM/TRRAP; FATC: FAT c-terminal; GATK: Genome Analysis Toolkit; Indel: Insertion/deletion; jMorp: Japanese Multi Omics Reference Panel; LZ: Leucine zipper; NBDC: National Bioscience Database Center; NLS: Nuclear localization signal; PI3K: Phosphoinositide 3-kinase; RR: Relative risk; SNP: Single-nucleotide polymorphism; SNV: Single-nucleotide variant; ToMMo: Tohoku Medical Megabank Organization

\section{Acknowledgments}

We would like to thank Prof. Kengo Kinoshita, Vice-President of ToMMo, for the helpful suggestions and comments.

\section{Authors' contributions}

HF contributed to the study design. HF, YT, and KMP analyzed the data. HF and KMP prepared the manuscript, table, and figures (all originals). All authors read and approved the final manuscript.

\section{Funding}

HF was founded by fellowships and research grants from the Marubun Research Promotion Foundation (FY2017-2018), the Japan Radiation Effects Association (FY2018). This work was supported by a research grant of the Japan Radiological Society from Bayer Yakuhin to HF.

\section{Availability of data and materials}

Please contact ToMMo (https://www.megabank.tohoku.ac.jp/english/) for additional information

\section{Ethics approval and consent to participate}

The present study was approved by the Institutional Review Board of ToMMo.

\section{Consent for publication}

A signed informed consent was obtained from each participant of ToMMo population-based biobank projects.

\section{Competing interests}

The authors declare that they have no competing interests.

\section{Author details}

'Centre for Cancer Research and Cell Biology, Queen's University Belfast, 97 Lisburn Road, Belfast BT9 7AE, UK. ${ }^{2}$ Shonan Kamakura General Hospital, 1370-1 Okamoto, Kamakura, Kanagawa 247-8533, Japan. ${ }^{3}$ Institute of Development, Aging and Cancer, Tohoku University, 4-1 Seiryo-machi, Aoba-ku, Sendai 980-8573, Japan. ${ }^{4}$ Tohoku Medical Megabank Organization, Tohoku University, 2-1 Seiryo-machi, Aoba-ku, Sendai 980-8575, Japan.

Received: 13 February 2019 Accepted: 19 August 2019

Published online: 23 August 2019

\section{References}

1. Paulino TL, Rafael MN, Hix S, Shiqueoka DC, Aizen SA, Kochi C, et al. Is age a risk factor for liver disease and metabolic alterations in ataxia telangiectasia patients? Orphanet J Rare Dis. 2017;12:136

2. Rothblum-Oviatt C, Wright J, Lefton-Greif MA, McGrath-Morrow SA, Crawford TO, Lederman HM. Ataxia telangiectasia: a review. Orphanet J Rare Dis. 2016;11:159.

3. Savitsky K, Bar-Shira A, Gilad S, Rotman G, Ziv Y, Vanagaite L, et al. A single ataxia telangiectasia gene with a product similar to PI-3 kinase. Science. 1995;268:1749-53.

4. Zhang N, Chen P, Khanna KK, Scott S, Gatei M, Kozlov S, et al. Isolation of full-length ATM CDNA and correction of the ataxia-telangiectasia cellular phenotype. Proc Natl Acad Sci U S A. 1997;94:8021-6.

5. Ambrose M, Gatti RA. Pathogenesis of ataxia-telangiectasia: the next generation of ATM functions. Blood. 2013;121:4036-45.

6. Bakkenist CJ, Kastan MB. DNA damage activates ATM through intermolecular autophosphorylation and dimer dissociation. Nature. 2003:421:499-506.
7. Kozlov SV, Graham ME, Peng C, Chen P, Robinson PJ, Lavin MF. Involvement of novel autophosphorylation sites in ATM activation. EMBO J. 2006;25:3504-14.

8. Kozlov SV, Graham ME, Jakob B, Tobias F, Kijas AW, Tanuji M, et al. Autophosphorylation and ATM activation: additional sites add to the complexity. J Biol Chem. 2011;286:9107-19.

9. Lee J-H, Paull TT. Activation and regulation of ATM kinase activity in response to DNA double-strand breaks. Oncogene. 2007;26:7741-8.

10. Swift M, Reitnauer PJ, Morrell D, Chase CL. Breast and other cancers in families with ataxia-telangiectasia. N Engl J Med. 1987;316:1289-94.

11. van Os NJH, Roeleveld N, Weemaes CMR, Jongmans MCJ, Janssens GO, Taylor AMR, et al. Health risks for ataxia-telangiectasia mutated heterozygotes: a systematic review, meta-analysis and evidence-based guideline. Clin Genet. 2016;90:105-17.

12. Jerzak KJ, Mancuso T, Eisen A. Ataxia-telangiectasia gene (ATM) mutation heterozygosity in breast cancer: a narrative review. Curr Oncol. 2018;25:e176-80.

13. Nagasaki M, Yasuda J, Katsuoka F, Nariai N, Kojima K, Kawai Y, et al. Rare variant discovery by deep whole-genome sequencing of 1,070 Japanese individuals. Nat Commun. 2015;6:8018.

14. Yamaguchi-Kabata Y, Nariai N, Kawai Y, Sato Y, Kojima K, Tateno M, et al. iJGVD: an integrative Japanese genome variation database based on wholegenome sequencing. Hum Genome Var. 2015;2:15050.

15. Yasuda J, Katsuoka F, Danjoh I, Kawai Y, Kojima K, Nagasaki M, et al. Regional genetic differences among Japanese populations and performance of genotype imputation using whole-genome reference panel of the Tohoku medical megabank project. BMC Genomics. 2018;19:551.

16. Tadaka S, Katsuoka F, Ueki M, Kojima K, Makino S, Saito S, et al. 3.5KJPNv2: an allele frequency panel of 3552 Japanese individuals including the $X$ chromosome. Hum Genome Var. 2019;6:28.

17. Tadaka S, Saigusa D, Motoike IN, Inoue J, Aoki Y, Shirota M, et al. jMorp: Japanese multi omics reference panel. Nucleic Acids Res. 2018:46:D551-7.

18. 1000 Genomes Project Consortium, Auton A, Brooks LD, Durbin RM, Garrison EP, Kang HM, et al. A global reference for human genetic variation. Nature. 2015:526:68-74.

19. Karczewski KJ, Francioli LC, Tiao G, Cummings BB, Alföldi J, Wang Q, et al. Variation across 141,456 human exomes and genomes reveals the spectrum of loss-of-function intolerance across human protein-coding genes. BioRxiv. 2019. https://doi.org/10.1101/531210.

20. Van der Auwera GA, Carneiro MO, Hartl C, Poplin R, del Angel G, LevyMoonshine A, et al. From FastQ data to high-confidence variant calls: the genome analysis toolkit best practices pipeline. Curr Protoc Bioinforma. 2013:43:11.10.1-33.

21. Sherry ST, Ward M, Sirotkin K. dbSNP-database for single nucleotide polymorphisms and other classes of minor genetic variation. Genome Res. 1999:9:677-9.

\section{Publisher's Note}

Springer Nature remains neutral with regard to jurisdictional claims in published maps and institutional affiliations.
Ready to submit your research? Choose BMC and benefit from:

- fast, convenient online submission

- thorough peer review by experienced researchers in your field

- rapid publication on acceptance

- support for research data, including large and complex data types

- gold Open Access which fosters wider collaboration and increased citations

- maximum visibility for your research: over $100 \mathrm{M}$ website views per year

At BMC, research is always in progress.

Learn more biomedcentral.com/submission 